DE

M E D I C I N A

T R O P I C A L

$\mathrm{DE}$

SÃO PAULO

JOURNAL OF THE SÃO PAULO INSTITUTE OF TROPICAL MEDICINE

${ }^{1}$ Instituto de Infectologia Emilio Ribas, São Paulo, São Paulo, Brazil

2Universidade de São Paulo, Faculdade de Medicina, Hospital das Clínicas, São Paulo, São Paulo, Brazil

3Universidade de São Paulo, Instituto de Medicina Tropical, São Paulo, São Paulo, Brazil

${ }^{4}$ Universidade Federal de São Paulo, São Paulo, São Paulo, Brazil

${ }^{5}$ Instituto Adolfo Lutz, São Paulo, São Paulo, Brazil

${ }^{6}$ Fundação Fiocruz, Instituto Nacional de Doenças Infecciosas, Rio de Janeiro, Rio de Janeiro, Brazil

${ }^{7}$ University of Minnesota, Minneapolis, Minnesota, United States of America

Correspondence to: José E. Vidal Universidade de São Paulo, Instituto de Medicina Tropical de São Paulo, Laboratório de Investigação Médica 49, Av. Dr. Enéas de Carvalho Aguiar, 470, CEP 05403-000, São Paulo, SP, Brazil

Tel/Fax: + $55113061-7010$

E-mail: josevibe@gmail.com

Received: 13 June 2017

Accepted: 16 October 2017

\section{Performance of cryptococcal antigen lateral flow assay in serum, cerebrospinal fluid, whole blood, and urine in HIV-infected patients with culture-proven cryptococcal meningitis admitted at a Brazilian referral center}

José E. Vidal'1,2,3, Carolina Toniolo', Adriana Paulino', Arnaldo L. Colombo4, Marilena dos Anjos Martins ${ }^{5}$, Cristina da Silva Meira ${ }^{5}$, Renata Guise Soares Azevedo1, Vera Lucia Pereira-Chioccola ${ }^{5}$, Hélio Rodrigues Gomes ${ }^{2}$, Marcia dos Santos Lazera ${ }^{6}$, Augusto C. Penalva de Oliveira1, David R. Boulware ${ }^{7}$

\section{ABSTRACT}

Cryptococcal meningitis is the most common cause of opportunistic meningitis in HIVinfected patients in Brazil and causes unacceptable high mortality rates. In this study, HIVinfected patients with a first episode of culture-proven cryptococcal meningitis in cerebrospinal fluid (CSF) were prospectively included in order to evaluate sensitivity of cryptococcal antigen $(\mathrm{CrAg})$ lateral flow assay (LFA) in serum, CSF, whole blood (fingerstick), and fresh urine. In addition, HIV-infected patients with other neurological confirmed diseases were included in order to evaluate the specificity of CrAg LFA in serum. Twenty patients with cryptococcal meningitis were included and in 19 of them, CrAg LFA in CSF, serum, and whole blood were positive ( $95 \%$ sensitivity). In 18 patients, India ink test was positive in CSF (90\% sensitivity), and in 16 cases, CrAg LFA was positive in urine (80\% sensitivity). Thirty-six HIV-infected patients with other neurological diseases had negative results of CrAg LFA in serum $(100 \%$ specificity). In conclusion, CrAg LFA in serum, CSF, and whole blood showed high sensitivity and specificity. Whole blood CrAg LFA seems to be a good and reliable strategy to improve AIDS-related cryptococcal meningitis diagnosis in Brazil.

KEYWORDS: Cryptococcal meningitis. Cryptococcosis. Diagnosis. Human immunodeficiency virus. Acquired immunodeficiency syndrome.

\section{INTRODUCTION}

The number of cases of cryptococcal meningitis diminished in the highly active antiretroviral therapy (HAART) era in Brazil. However, Cryptococcus neoformans continues to be the most common cause of opportunistic meningitis in our setting and causes high lethality rates ${ }^{1}$. The World Health Organization (WHO) recommends the use of cryptococcal antigen $(\mathrm{CrAg})$ assays by latex agglutination or lateral flow assay (LFA) as the preferred diagnostic approaches for cryptococcal meningitis diagnosis in resource-limited settings ${ }^{2}$. CrAg LFA (Immuno-Mycologics, Norman, OK, USA) is an immunochromatographic assay that can be used in settings with minimal or no infrastructure. CrAg LFA is the only commercially available $\mathrm{CrAg}$ assay that complies with WHO ASSURED criteria (Affordable, Sensitive, Specific, User friendly, Rapid/robust, Equipment-free, and Delivered $)^{3}$ for diagnostic tests. In addition, CrAg LFA has better analytical sensitivity than other commercial assays for both $C$. neoformans and C. gatti ${ }^{4}$. The CrAg LFA has been approved 
by the U.S. Food and Drug Administration (FDA) for use in serum and cerebrospinal fluid (CSF) specimens, and similar use has recently been released in Brazil. However, unvalidated specimen types of whole blood or urine constitute potentially ideal samples for point-of-care testing of individual patients. The diagnostic performance in urine or whole blood is less clear.

In this validation study, we evaluated the performance of CrAg LFA in HIV-infected patients with cryptococcal meningitis.

\section{PATIENTS AND METHODS}

\section{Group 1: Cryptococcal meningitis}

Twenty HIV-infected patients in their first episode of cryptococcal meningitis confirmed by CSF fungal culture were included in order to evaluate the sensitivity of $\mathrm{CrAg}$ LFA in serum, CSF, whole blood (fingerstick) and fresh urine samples. For CSF and serum samples, manufacturer's instructions were followed, and for whole blood and urine, already established protocols ${ }^{5,6}$. In addition, cerebrospinal India Ink and fungal blood culture were obtained in all cases, at admission. All patients received amphotericin B deoxycholate $(0.7$ to $1.0 \mathrm{mg} / \mathrm{kg} /$ day, intravenously) and fluconazol $(800 \mathrm{mg} /$ day, orally in two divided doses) or flucytosine (100 mg/kg/day, orally, in four divided doses) were associated for at least two weeks, in 18 and 2 cases, respectively. Subsequently, fluconazole (400-800 mg per day, orally) was prescribed for at least eight weeks. Nine $(45 \%)$ patients had intracranial hypertension at diagnosis with CSF opening pressure of $25 \mathrm{cmH}_{2} \mathrm{O}$, requiring serial lumbar punctures. In four (20\%) cases, neurosurgery was performed and a lumbar peritoneal shunt was placed in order to control intracranial pressure.

\section{Group 2: HIV controls}

Thirty-six HIV-infected patients with other confirmed neurological diseases were included in order to evaluate the specificity of CrAg LFA in serum. Sensitivity was defined as the probability that a test result will be positive when the disease is present (true positive rate). Specificity was defined as the probability that a test result will be negative when the disease is not present (true negative rate). In addition, a 95\% confidence interval $(95 \% \mathrm{CI}$ ) was calculated.

Participants were prospectively recruited over a period of one year at the Emilio Ribas Institute of Infectious Diseases, São Paulo, Brazil. The study was conceived in the Cryptococcosis Brazilian Network, which provided the CrAg LFA kits, and was approved by the Research Ethics
Committee of the hospital (Research Protocol No 46/2014). All study participants provided written informed consent. Analysis was primarily descriptive reporting the diagnostic performance.

\section{RESULTS}

\section{Group 1: Cryptococccal meningitis}

The mean age $( \pm \mathrm{SD})$ was $39.9( \pm 6.2)$ years old, and 12 subjects $(60 \%)$ were men. Cryptococcal meningitis was the initial AIDS-definition disease in $11(55 \%)$ cases. Fifteen (75\%) patients were HAART experienced; however, none of them was under medications at admission. The CD4 cell count median was 42 (Range: 1 - 176) cells/ $\mu \mathrm{L}$. Among the 20 patients, the first lumbar puncture demonstrated culture-proven diagnosis of cryptococcal meningitis in 18 cases. In the other 2 patients, cultureproven diagnosis was obtained from the second lumbar puncture. In all 20 cases, C. neoformans was identified. Among the 20 patients, 19 had positive CrAg LFA in CSF, serum, and whole blood samples (95\% sensitivity; $95 \% \mathrm{CI}$ : $75.13 \%$ to $99.87 \%)$. In 18 patients (90\%), CSF India ink was positive at admission. In one case, the single positive test was CSF fungal culture. This case was one of the two with proven diagnosis by the culture in the second lumbar puncture, performed one day after the first one. The false negative result of CrAg LFA was perfomed with the sample of the second lumbar puncture. Sixteen $(80 \%)$ patients had positive CrAg LFA in urine; in six patients (32\%), C. neoformans growing was observed in blood culture. Two (10\%) patients died during hospitalization, and 18 (90\%) were discharged home.

\section{Group 2: HIV controls}

In all thirty-six controls, serum CrAg LFA was negative (100\% specificity; $95 \%$ CI: $90.3 \%$ to $100.0 \%$ ). Among them, 18 controls with tuberculous meningitis; 9 controls with neurosyphilis; 5 controls with progressive multifocal leukoencephalopathy; 3 controls with cytomegalic encephalitis; and 1 control with cerebral toxoplasmosis.

\section{DISCUSSION}

Our prospective study reinforces the diagnostic value of serum and CSF CrAg LFA testing in patients with cryptococcal meningitis. A systematic review showed a pooled sensitivity of serum CrAg LFA of $97.6 \%$ (95\% CI, 95.6\% to $98.9 \%$ ) and CSF CrAg LFA of $98.9 \%$ (95\% CI, $97.9 \%$ to $99.5 \%$ ). In addition, this review pointed 
out a pooled specificity of serum CrAg LFA of $98.1 \%$ (95\% CI, $97.4 \%$ to $98.6 \%$ ) and CSF CrAg LFA of $98.9 \%$ (95\% CI, $98 \%$ to $99.5 \%)^{7}$. More interestingly, we found $100 \%$ agreement between whole blood and serum $\mathrm{CrAg}$ LFA results, as previously reported ${ }^{5}$. Thus, although it is not a FDA approved specimen, whole blood or plasma are a reliable and practical fluid for CrAg LFA tool for detection of $\mathrm{CrAg}$ as a point-of-care test. Our results have also emphasized that urine has limitations given its pooled sensitivity of $85.0 \%$ (95\% CI, $78.7 \%$ to $90.1 \%)^{7}$.

CrAg LFA implementation constitutes a great opportunity to optimize diagnosis time and resources in settings where cryptococcal meningitis is a main cause of opportunistic meningitis in HIV-infected persons. Nowadays, in several hospital referral centers in Brazil, when a first CSF is collected from an HIV-infected patient with clinical suspicion of meningitis or meningoencephalitis, several tests are requested, including most of the following tests: Gram's stain; acid-fast bacillus (AFB) smear; bacterial, fungal and mycobacterial cultures; pan-Herpes PCR; nucleic acid amplification tests for tuberculosis and a $\mathrm{CrAg}$ latex agglutination assay. On the other hand, in most centers with lesser resources, it is relevant to strategically identify which tests are the most relevant to diagnosis. As recently proposed, development of algorithms using a fingerstick CrAg LFA as an initial test seems to be more rational for investigating meningitis or meningoencephalitis in HIV-infected patients ${ }^{5}$.

Despite the advantages of CrAg LFA versus other tests, there are several barriers to incorporate this tool into routine testing. For example, the price of each test in São Paulo is approximately US\$ 9.5 (Interlab - Distribuidora de Produtos Cientificos Ltda, in March the $6^{\text {th }}, 2017$ ), similar to the price of the most popular local CrAg latex agglutination assay. This price is two-fold higher than purchase price in the United States of America.

In conclusion, CrAg LFA in serum, CSF and whole blood showed high sensitivity and specificity. Whole blood CrAg LFA seems to be a simple strategy to improve diagnosis of AIDS-related cryptococcal meningitis in Brazil. Despite current barriers, implementation of this tool could reduce costs by streamlining diagnostic testing.

\section{REFERENCES}

1. Vidal JE, Penalva de Oliveira AC, Dauar RF, Boulware DR. Strategies to reduce mortality and morbidity due to AIDSrelated cryptococcal meningitis in Latin America. Braz J Infect Dis. 2013;17:353-62.

2. World Health Organization. Rapid advice: diagnosis, prevention and management of cryptococcal disease in HIV-infected adults, adolescents and children. Geneva: WHO; 2011.

3. Kozel TR, Bauman SK. CrAg lateral flow assay for cryptococcosis. Expert Opin Med Diagn. 2012;6:245-51.

4. Vidal JE, Boulware DR. Lateral flow assay for cryptococcal antigen: an important advance to improve the continuum of HIV care and reduce cryptococcal meningitis-related mortality. Rev Inst Med Trop Sao Paulo. 2015;57 Suppl 19:38-45.

5. Williams DA, Kiiza T, Kwizera R, Kiggundu R, Velamakanni $\mathrm{S}$, Meya DB, et al. Evaluation of fingerstick cryptococcal antigen lateral flow assay in HIV-infected persons: a diagnostic accuracy study. Clin Infect Dis. 2015;61:464-7.

6. Magambo KA, Kalluvya SE, Kapoor SW, Seni J, Chofle AA, Fitzgerald DW, et al. Utility of urine and serum lateral flow assays to determine the prevalence and predictors of cryptococcal antigenemia in HIV-positive outpatients beginning antiretroviral therapy in Mwanza, Tanzania. J Int AIDS Soc. 2014;17:19040.

7. Huang HR, Fan LC, Rajbanshi B, Xu JF. Evaluation of a new cryptococcal antigen lateral flow immunoassay in serum, cerebrospinal fluid and urine for the diagnosis of cryptococcosis: a meta-analysis and systematic review. PLoS One. 2015;10:e127117. 